Original article

\title{
Antimicrobial susceptibility of Streptococcus pneumoniae isolates causing LRTI in
}

Najaf, Iraq

\author{
Zahraa Y. Motaweq ${ }^{1}$, Habeeb S. Naher ${ }^{2}$ \\ ${ }^{1}$ Department of Biology, College of Science, Kufa University \\ ${ }^{2}$ Department of Microbiology, College of Medicine, Babylon University \\ E-mail address: muhaimin.alrufaie@uokufa.edu.iq
}

\begin{abstract}
During the period from February 2013 to April 2014, 74 (12.3\%) isolates of Streptococcus pneumoniae were isolated from 600 patients (359 males and 241 females) with clinical symptoms of Lower respiratory tract infections (LRTI) (pneumonia and COPD) obtained from Najaf/Iraq Hospitals. Patients in the age groups 51-60 years had a high percentage of $S$. pneumoniae isolates $(19.7 \%)$ compared with other age groups with a significant variation $(\mathrm{P}<0.05)$ between them. Males $(54 \%)$ showed a higher percentage of $S$. pneumoniae isolates than females (45.9\%) with no significant variation (P>0.05). Smokers have been shown to have increased risk to LRTI than non-smokers ( $>0.05)$, and there was no significant variation between Urban and Rural (56.8:43.2\%) patients. S. pneumoniae showed different susceptibilities towards antibiotics used in this study. The highest rate of resistance was against erythromycin (100\%), azithromycin (83.8\%), clindamycin (83.8\%) and trimethoprim/sulfamethaxzol $(81.1 \%)$ and moderate resistance to ceftriaxone (67.6\%), cefotaxime (64.9\%), chloramphenicol (64.9\%), tetracycline (59.5\%) and benzylpenicillin (45.9\%) whereas there was a relatively lower resistance towards others. The results of this study showed that S. pneumoniae isolates were found to be remarkable sensitive to Vancomycin (100\%) and Imipenem (100\%). In this study, sixteen antibiotics were tested for (MIC) against 37 S. pneumoniae isolates by using Vitek-2 antibiotic susceptibility testing (AST) cards (41497) AST-GP74. 100\% and 83.8\% of S. pneumoniae isolates were resistant to erythromycin and SXT with MIC $\geq 1 \mathrm{mg} / \mathrm{ml}$ and $4 / 76 \mathrm{mg} / \mathrm{ml}$ of these antibiotic respectively, and moderately resistant to cefotaxime $64.9 \%$, ceftriaxone $64.9 \%$ and chloramphenicol $64.9 \%$ with MIC $4 \mathrm{mg} / \mathrm{ml}$ for CTX and CRO each one, and MIC $8 \mathrm{mg} / \mathrm{ml}$ for C only. All isolates showed $100 \%$ sensitivity for each of Vancomycin and Erythromycin with MIC $\mathrm{mg} / \mathrm{ml}$ and $\leq 1 \mathrm{mg} / \mathrm{ml} \mathrm{and} \leq 2 \mathrm{mg} / \mathrm{ml}$, respectively. S. pneumoniae isolates showed a high rate of sensitivity to Ertapenem $97.3 \%$ with MIC $\leq 1 \mathrm{mg} / \mathrm{ml}$, Telithromycin $89.2 \%$ with MIC $\leq 1$, Meropenem $86.5 \%$ with MIC $\leq 0.25 \mathrm{mg} / \mathrm{ml}$.
\end{abstract}

KEY WORDS: Streptococcus pneumoniae, antibiotic resistance, lower respiratory tract infections (LRTI), Minimum Inhibitory Concentration (MIC), COPD

ARTICLE HISTORY: received 6 January 2017; accepted 5 June 2017

\section{Introduction}

S. pneumoniae is the major cause of communityacquired pneumonia in adults and serious respiratory infections in children in the United States. An estimated three to five million deaths occur annually in children under 5 years of age due to acute respiratory infections, for which $S$. pneumoniae is the most important pathogen (OBARO, 2000). The incidence of pneumococcal disease and the occurrence of antibiotic resistant isolates have been positively correlated to the levels of carriage (PAUL, 1997). Carriage can be influenced by age (highest in infants and decreasing with age), immune status, seasonal variation, socio-economic factors, and other demographic features (GRAY ET AL., 1982).

Penicillin is the antimicrobial agent of choice, and macrolides are the second most common alternative. Within the last two decades, the emergence of strains of $S$. pneumoniae that are resistant to penicillin, macrolides, and other antimicrobial agents has become a serious health care problem (JORGENSEN ET AL., 2004). The present study was designed to isolate and identify S. pneumoniae from LRTI patients, by using conventional and Vitek-2 
techniques and studying the antibiotic susceptibility of $S$. pneumoniae to different antibiotics by disk diffusion methods DDM and MICs.

\section{Material and methods}

Patients and clinical specimens. A total of 600 sputum samples were collected from out- and inpatients who were suffering from lower respiratory tract infections (LRTI) (pneumonia, COPD) attending at the Chest Unit in Al-Sadder Medical City, AlHakeem General Hospital and Clinic Consultive Center for Chest Disease and Al-Zahra'a Hospital for Childbirth and Children in Al-Najaf province during the period from February 2013-April 2014. The patients included both sexes and were in the age range 1-80 years.

Bacterial isolates and culture conditions. Quantitative sputum cultures were made for each specimen according to sputum gram stain for pneumonia infections. Sputum specimens were homogenized with an equal volume of normal saline on a vortex mixer. Blood agar and Chocolate agar were inoculated with $0.1 \mathrm{ml}$ of homogenized specimen and spread on the plates with a sterile loop. Plates were incubated in 5-10\% $\mathrm{CO}_{2}$ candle jar at $37^{\circ} \mathrm{C}$ for overnight (WILSON \& MARTIN, 1972). The plates were examined thereafter for bacterial growth and positive plates ( $\alpha$-haemolysis). A single pure isolated colony was transferred to trypticase soy agar for preservation and was to submitted to morphological evaluation by Gram staining and other biochemical tests were carried to confirm the identification of isolates of S. pneumoniae.

Identification of Bacteria. The identification of S. pneumoniae was achieved according to cellular morphology, culture characters and biochemical reactions as in MACFADDIN (2000).

Optochin Test. A half plate of 5\% sheep blood agar was streaked with an inoculum from a pure isolate of the organism to be tested, then an optochin disc was placed in the center of the inoculum and was incubated for $24-48$ hours at $37^{\circ} \mathrm{C}$ in a candle jar, then an observation of a zone of growth inhibition greater than $14 \mathrm{~mm}$ surrounding the disc was considered as positive. This test was used to differentiate $S$. pneumoniae (sensitive) from viridans streptococci (resistance) (CollEE ET AL., 1996).

Bile Solubility Test. There are two methods for this test, tube method and plate method (MACFADDIN, 2000).

Inulin Fermentation Method. The isolates were inoculated into tubes of phenol red inulin broth and incubated at $37^{\circ} \mathrm{C}$ for $24 \mathrm{hrs}$. The tubes were then examined for the presence of a yellow color indicative of acid formation from the fermentation of inulin and red indicates no inulin fermentation tube (MACFADDIN, 2000).

Identification of Bacteria by STREPTO-SYSTEM 9R Kit. STREPTO-SYSTEM 9R for S. pneumoniae identification was used according to the recommendation of the company (Liofilchem, England).

Vitek-2 for Identification. A GP identification card was used for the identification of pneumococci (Guido \& PASCALE, 2005).

Antibiotic Susceptibility Testing.

Disk Diffusion Method. This test was performed according to the instructions of the Clinical Laboratory Standards Institute (CLSI, 2014) by using a pure culture of the previously identified bacterial organism. A suspension of 5 isolated colonies was grown on blood agar plates of $5 \mathrm{ml}$ of tryptic soy broth. This culture was then incubated for 2 hrs to produce a bacterial suspension of moderate turbidity and was compared with the turbidity of a ready-made 0.5 McFarland standard tube. A sterile swab was used to obtain an inoculum from the standardized culture, this inoculum was then swabbed on a Muller-Hinton agar plate supplemented with $5 \%$ horse blood and the antibiotic discs were placed on the surface of this medium and incubated at $37^{\circ} \mathrm{C}$ for $24 \mathrm{hrs}$. Antibiotic inhibition zones were measured and zone size was compared with standard zones from the CLSI (2014), to determine the susceptibility of the organism to each antibiotic.

Vitek-2 for Antimicrobial Susceptibility. An antimicrobial susceptibility test was also carried out using the Vitek-2 system (bioMérieux, France), by utilising AST-P79 Gram positive susceptibility cards. The antibiotics tested included cephalosporine (cefotaxime, ceftriaxone), benzylpenicillin, aminopenicillin (amoxicillin), tetracycline, chloramphenicol as well as quinolones (levofloxacin, moxifloxacin), macrolide (erythromycin, telithromycin), linezolid, carbapeneme (ertapenem, meropenem), ofloxacin, sulfonamides (trimethoprim/ sulfamethaxol) and glycopeptides (vancomycin).

\section{Results and discussion}

\subsection{Prevalence of $S$. pneumoniae according to risk factors}

In this study, the prevalence of $S$. pneumoniae isolates based on patient in different age groups 
are shown in Fig. 1. The highest prevalence appeared in the 51-60 years age group at $19.7 \%$, followed by others at age groups over 60 years, 31-40 years and $1-10$ years at $14 \%, 11 \%$ and $10.8 \%$, respectively. A low isolation frequency was recorded in $11-20$ years and $21-30$ years with $5.1 \%$ and $3.8 \%$ respectively, i.e. the youth period. There was no significant difference $(\mathrm{P}>0.05)$ according to sex, between pneumococcus isolates and sex. However, more $S$. pneumoniae were isolated from males (54.1\%) than females (45.9\%) (Fig. 2). There was no significant difference $(\mathrm{P}>0.05)$ between place of residence and pneumococcal infection (Fig. 3). This could be attributed to the fact that smoking is more common in males and Fig. 4 shows the incidence of pneumonia in patients who are smokers have the same percent (54.1\%) as males with a pneumococcal infection. Smoking has been shown to substantially increase the risk of invasive pneumococcal disease.

These results are in agreement with AL-TAAIE (2013), who reported that pneumococci showed a total incidence in those aged over 49 years, despite the differences in percent of isolates. PEJCIC ET AL., (2011) observed pneumococcal pneumonia is much more frequent in elderly people than in younger and middle-aged populations. GUPTA ET AL, (2012) reported that pneumococcus is the single most common organism identified in hospitalized elderly patients with CAP. Worldwide the pneumococcus is responsible for more than 14.5 million episodes of IPD annually and up to $11 \%$ of all deaths in children (O'BRIEN ET AL, 2009). Notably, in individuals over the age of 65 the case-fatality rate for IPD can be as high as 30\% (MARUYAMA ET AL., 2010). Thus pneumococcal infections are a major medical problem for both children and the elderly.

The high incidence of the pneumococcal isolate in the 51-60 age group may be due to impairement of the immune system, and most elderly people are infected with chronic diseases. In the age group 31-40, females (8) had a higher number of case than males (2), most females during this age group may be taking many drugs and hormones for pregnancy difficulties, so that they may be exposed to infection due to a decrease in their immunity. The low immunity and higher exposure to contaminated materials may have a role in increased infection rates with pneumonia in the 1-10 age group.

Pneumonia remains the leading cause of mortality from infectious disease worldwide. In 2008 alone, it killed nearly 1.6 million children $<5$ years of age (BLACK ET AL., 2010). Over 90\% of the estimated 1.8 million annual deaths due to acute respiratory infections in children less than 5 years of age occur in developing countries and are mainly due to bacterial infections (BRYCE ET AL., 2005). Typical bacterial pathogens causing CAP in children less than 5 years of age include $S$. pneumoniae, Haemophilus influenzae, and Mycoplasma pneumoniae (GENTILE ET AL., 2012).

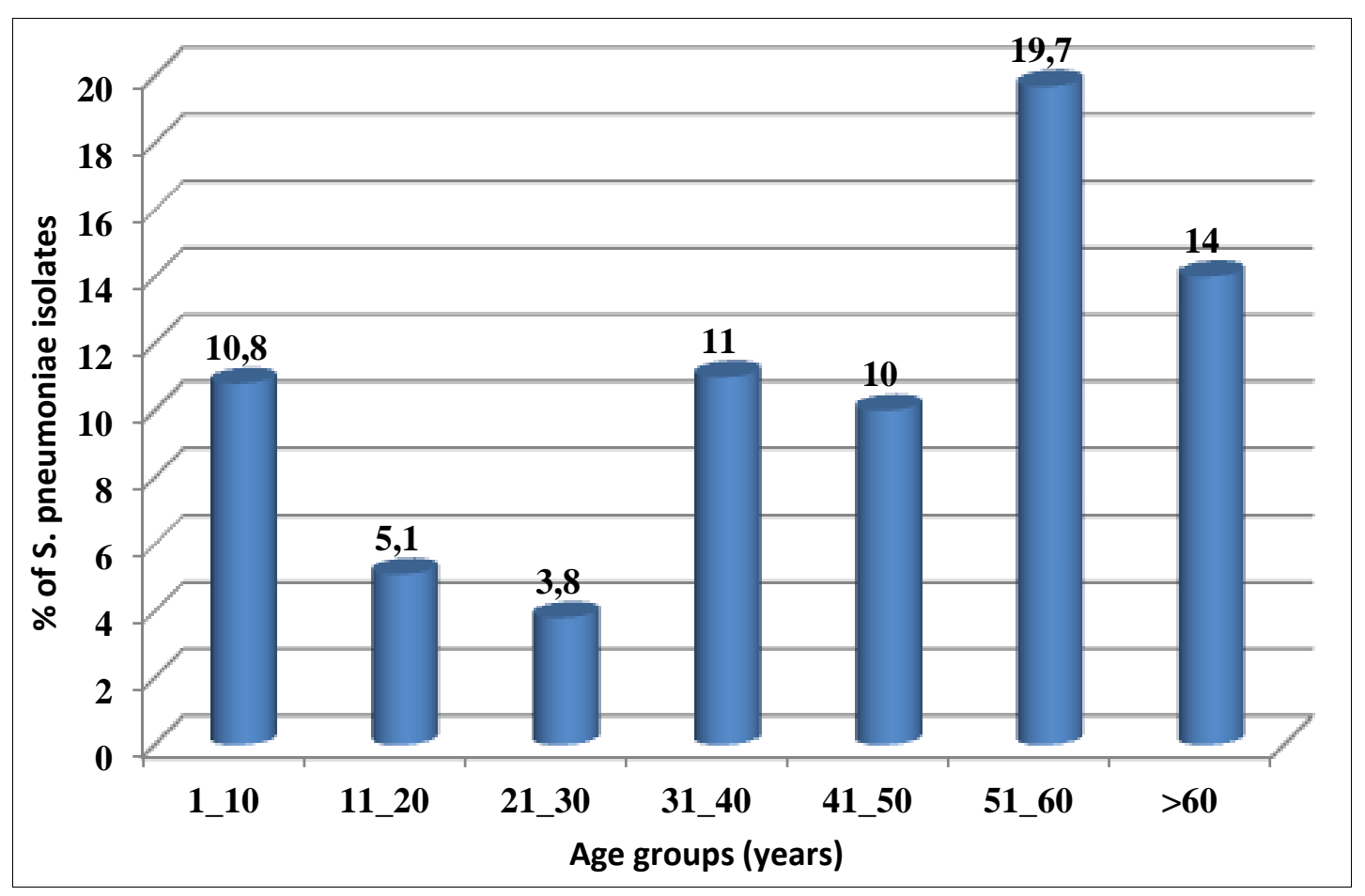

Fig. 1. Prevalence of S. pneumoniae according to age 


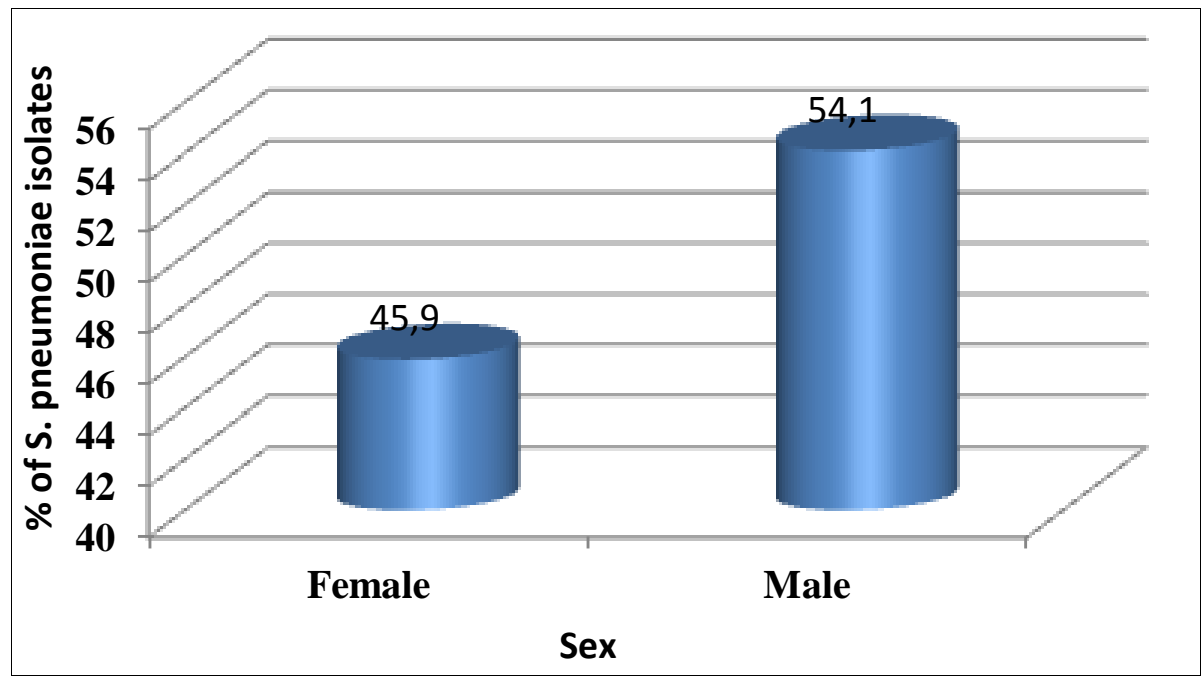

Fig. 2. Prevalence of S. pneumoniae according to sex

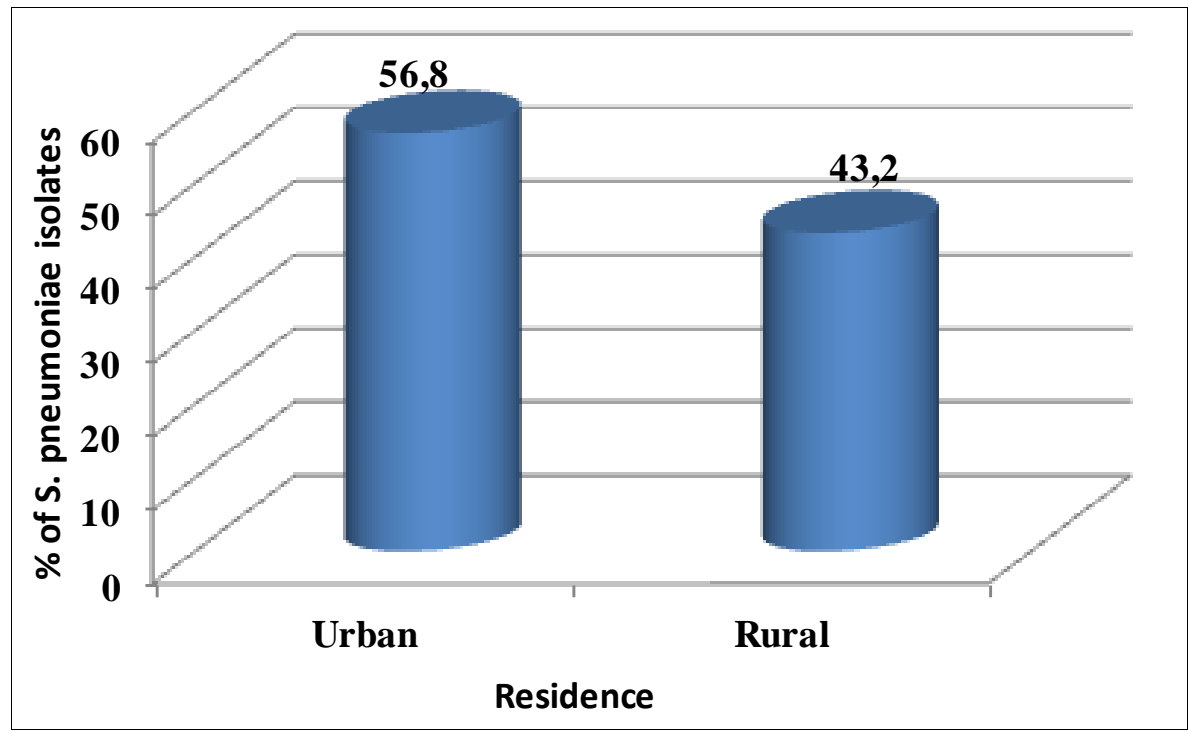

Fig. 3. Prevalence of S. pneumoniae according to place of residence

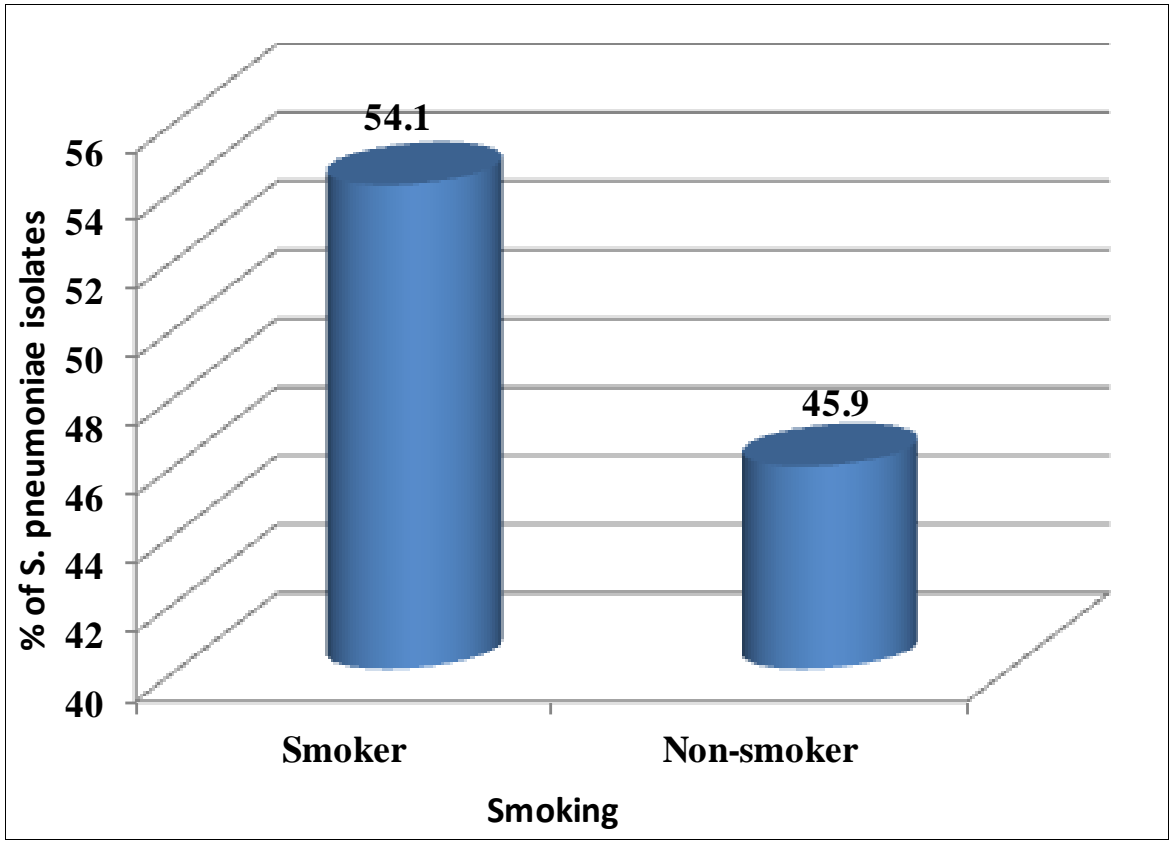

Fig. 4. Prevalence of S. pneumoniae in smokers and non-smokers 


\subsection{Antimicrobial Susceptibility test}

Antibiotic sensitivity test. Disk diffusion method (DDT). Fig. 5 and Table 1 show the phenotypic susceptibility of 37 S. pneumoniae isolates to 24 commonly used antimicrobial agents by using the Kirby-Bauer disk diffusion method (BAUER ET AL., 1966). The results were interpreted according to the diameter of the inhibition zone and compared with standard zones of inhibition determined by CLSI (2014).

The results of this test showed that $S$. pneumoniae has a great resistance to most commonly antibiotics used in hospitals, S. pneumoniae showed different susceptibilities towards antibiotics used in this study (Fig. 5). The highest rate of resistance is seen with erythromycin $37 / 37$ (100\%), azithromycin $31 / 37$ (83.8\%), clindamycin 31/37 (83.8\%) and trimethoprim/sulfamethaxzol $30 / 37(81.1 \%)$ and moderate resistance to ceftriaxone $25 / 37$ (67.6\%), cefotaxime 24/37 (64.9\%), chloramphenicol 24/37 (64.9\%), tetracycline $22 / 37$ (59.5\%) and benzylpenicillin 17/37 (45.9\%), whereas relative lower resistance towards amoxicillin 13/37 (35.1\%), levofloxacin 13/37 (35.1\%), amoxicillin-clavulanic acid 12/37 (32.4\%), cefoxitin 12/37 (32.4\%), meropenem 6/37 (16.2\%), ciprofloxacin 6/37 (16.2\%), telithromycin 5/37 (13.5\%), gentamicin 4/37 (10.8\%), clarithromycin 4/37 (10.8\%), cefepime $3 / 37$ (8.1\%), oxacillin $3 / 37$ (8.1\%), rifampicin 3/37 (8.1\%) and ertapenem 1/37 (2.7\%). Development of antibiotic resistance is often related to the overuse, and misuse, of the antibiotics prescribed. Resistance of $S$. pneumoniae continues to be an important clinical therapeutic problem, such that there is an increasing multidrug resistance in this bacteria. The results of this study show that $S$. pneumoniae isolates were found to be remarkably sensitive to Vancomycin $(100 \%)$ and Imipenem (100\%).

The resistance rate of $S$. pneumoniae varies with the locality (region) studied, and is influenced by the frequency and intensity of utilization, and empirical use of the antimicrobial drugs (GOSSENS, 2009).

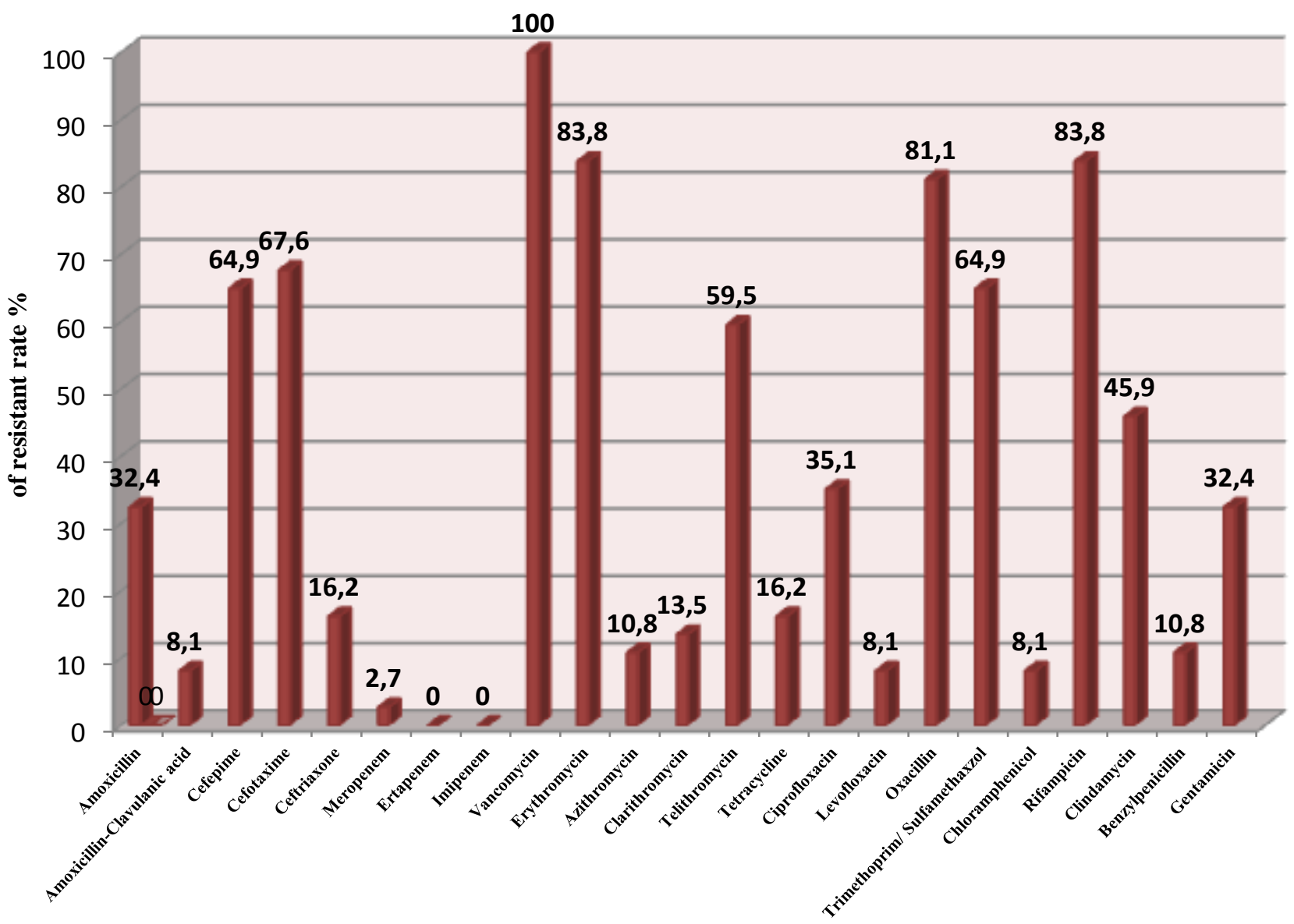

Antibiotics

Fig. 5. Resistance rate of $S$. pneumoniae isolates to 23 antibiotics 
Table 1. Antibiogram of 37 S. pneumoniae isolates

\begin{tabular}{|c|c|c|c|c|c|c|c|}
\hline \multirow[t]{2}{*}{ Antibiotics } & \multirow[t]{2}{*}{ Symbol } & \multicolumn{2}{|c|}{$S$} & \multicolumn{2}{|c|}{ I } & \multicolumn{2}{|c|}{$\mathrm{R}$} \\
\hline & & No. & $(\%)$ & No. & $(\%)$ & No. & $(\%)$ \\
\hline Amoxicillin & $\mathrm{Ax}$ & 24 & 64.9 & 0 & 0 & 13 & 35.1 \\
\hline Amoxicillin-Clavulanic acid & AMC & 21 & 56.8 & 4 & 10.8 & 12 & 32.4 \\
\hline Cefepime & FEP & 32 & 86.5 & 2 & 5.4 & 3 & 8.1 \\
\hline Cefotaxime & CTX & 13 & 35.1 & 0 & 0 & 24 & 64.9 \\
\hline Ceftriaxone & CRO & 12 & 32.4 & 0 & 0 & 25 & 67.6 \\
\hline Meropenem & MEM & 31 & 83.8 & 0 & 0 & 6 & 16.2 \\
\hline Ertapenem & ETP & 36 & 97.3 & 0 & 0 & 1 & 2.7 \\
\hline Imipenem & IPM & 37 & 100 & 0 & 0 & 0 & 0 \\
\hline Vancomycin & VA & 37 & 100 & 0 & 0 & 0 & 0 \\
\hline Erythromycin & $\mathrm{E}$ & 0 & 0 & 0 & 0 & 37 & 100 \\
\hline Azithromycin & AZM & 6 & 16.2 & 0 & 0 & 31 & 83.8 \\
\hline Clarithromycin & CLR & 27 & 73 & 6 & 16.2 & 4 & 10.8 \\
\hline Telithromycin & TEL & 32 & 86.5 & 0 & 0 & 5 & 13.5 \\
\hline Tetracycline & $\mathrm{TE}$ & 14 & 37.8 & 1 & 2.7 & 22 & 59.5 \\
\hline Ciprofloxacin & CIP & 27 & 73 & 4 & 10.8 & 6 & 16.2 \\
\hline Levofloxacin & LEV & 22 & 59.5 & 2 & 5.4 & 13 & 35.1 \\
\hline Oxacillin & OX & 31 & 83.8 & 3 & 8.1 & 3 & 8.1 \\
\hline Trimethoprim/Sulfamethaxzol & SXT & 7 & 18.9 & 0 & 0 & 30 & 81.1 \\
\hline Chloramphenicol & $\mathrm{C}$ & 12 & 32.4 & 1 & 2.7 & 24 & 64.9 \\
\hline Rifampicin & $\mathrm{RA}$ & 28 & 75.7 & 6 & 16.2 & 3 & 8.1 \\
\hline Clindamycin & DA & 6 & 16.2 & 0 & 0 & 31 & 83.8 \\
\hline Benzylpenicillin & $\mathrm{P}$ & 7 & 18.9 & 13 & 35.1 & 17 & 45.9 \\
\hline Gentamicin & $\mathrm{CN}$ & 29 & 78.4 & 4 & 10.8 & 4 & 10.8 \\
\hline Cefoxitin & FOX & 16 & 43.24 & 3 & 8.1 & 12 & 32.4 \\
\hline
\end{tabular}

FLAMM ET AL. (2013) found that S. pneumoniae was evaluated for MDR status against PEN, CRO, erythromycin (ERY), tetracycline (TET), trimethoprim/sulfamethoxazole (TMP/SMX), and levofloxacin (LEV). MDR were defined as nonsusceptible (NS) to at least 2 of the above agents. Ceftaroline and levofloxacin exhibited high rates of susceptibility at 100.0 and $98.9 \%$, respectively. Ceftriaxone, cefuroxime and amoxicillin/clavulanate susceptibilities were 89.2, 71.8 and 83.3\%, respectively. There was a high rate of resistance to erythromycin at $42.7 \%$, and resistance to tetracycline, trimethoprim/sulfamethoxazole and clindamycin ranged from 20.3 to $24.6 \%$. The results of this study disagrees with the FLAMM ET AL. (2103) study since, the $S$. pneumoniae isolates in this study exhibited a low rate of susceptibility to levofloxacin and other antibiotics, while showing $100 \%$ resistance to erythromycin.

On the other hand, during 2000-2001, there was a reported rise in resistance rates to fluoroquinolones FQ from invasive $S$. pneumoniae reaching up to $3.5 \%$ of isolates (SENER ET AL.,
2007). In Lebanon, S. pneumoniae isolates have shown increasing resistance to penicillin, macrolides, and other antimicrobial agents, but to our knowledge, none so far have been reported against FQ (KANJ ET AL., 2007).

RIJAL ET AL. (2010) have pointed out that 2.17\% of $S$. pneumoniae isolates were resistant to erythromycin and chloramphenicol and $2.17 \%$ of isolates were intermediately resistant to cefotaxime. This result was in imputable with results of the present study in which $100 \%$ of isolates appeared to be resistant to erythromycin an $64.9 \%$ for chloramphenichole and cefotaxim .

The new fluoroquinolones are widely used to treat respiratory tract infections, especially in patients with COPD. Although DOMENECH ET AL. (2011) showed a low rate $3.5 \%$ of ciprofloxacin resistance in pneumonia isolates; it was lower than rates found in a recent study $16.2 \%$. The high consumption of quinolones could explain the higher resistance rate. The differences observed in the rates of susceptibility to ciprofloxacin $16.2 \%$ and to levofloxacin $35.12 \%$ are due to 
isolates with first-step mutations in the quinolone resistance-determining regions. These isolates (ciprofloxacin susceptible and levofloxacin resistant) may become highly resistant under selective fluoroquinolone pressure and are associated with treatment failure when quinolones are used (FULLER \& LOW, 2005). The association trimethoprim/ sulphamethoxazole used this study showed a high rate of resistance, $81 \%$ full resistance. Compared with a preceding study with strains in Brazil, where there was an increased number of resistant strains, similar to the situation in other countries (RIEDEL ET AL., 2007) and the combination of trimethoprim/sulphamethoxazole should not be recommended to treat pneumococcal infections, because of the high rate of resistant strains.

LEVIN ET AL. (1996) observed a rate of tetracycline resistance as opposed to that reported in this study (32\%). $14 \%$ of strains showed intermediate resistance, and $4 \%$ of strains showed full resistance. Of these tetracycline-resistant strains, were multiresistant. These results indicate that the empirical use of tetracycline in pneumococcal infections is limited. WEBER ET AL. (2010) observed a rate of chloramphenicol, where $3 \%$ of the strains were resistant. All isolates showed full resistance to erythromycin.

The emergence of high-level resistance to antimicrobials is an increasing threat to global health (LEVY \& MARSHALL, 2004), and even a small increase in antibiotic-refractory bacterial subpopulations or MIC could herald the emergence of higher-level resistance (BALABAN ET AL., 2004). Therefore, any factor contributing to an increase of antibiotic resistance is critically important. TRAN ET AL. (2011) found that penicillin-triggered lysis could be partially prevented by heat shock pre-treatment and makes clear that in vivo stresses, such as inflammation, respiratory bursts in phagocytes, and temperature upshift, may induce higher ClpL levels and increase resistance to penicillin.

The most notable finding of this study is the level of erythromycin resistance $(100 \%)$ and the result was in accordance with the findings. Currently, the antibiotic resistance patterns of $S$. pneumoniae isolates vary widely from one country to another within Europe. Studies have shown wide variations of antimicrobial resistance against $S$. pneumoniae in different countries. Chloramphenicol and Erythromycin resistance was observed in only $2.17 \%$ of the strains without any relationship to Penicillin resistance. These drugs are the most common regime used in the hospitals in Iraq, and this is still a good empirical choice for the treatment of pneumoccocal meningitis.

Antibiotic sensitivity test. Minimum Inhibitory Concentration (MIC) Test. To confirm the results obtained by DDT this study used the MIC method for testing the susceptibility of the S. pneumoniae towards some tested antibiotics in an attempt to detect the proper method and to give an indication of the situation of the susceptibility of this organism to these antibiotics. The MIC values were based on the break point recommended by CLSI (2014) for estimation of the response. The break point represents the optimum concentration of the drug that can reach the serum and provide a high level of therapy. The micro-organism was considered sensitive if the estimated MIC was less than the break point. In the present study, sixteen antibiotics were tested for MIC against 37 S. pneumoniae isolates by using Vitek-2 Gram positive (GP) antibiotics susceptibility testing (AST) cards (41497) AST-GP74 (Biomer)(Table 2).

The results of the antibiogram test with the automated Vitek- 2 compact system revealed that $100 \%$ and $83.8 \%$ of $S$. pneumoniae isolates were resistant to erythroycin and SXT with MIC $\geq 1$ $\mathrm{mg} / \mathrm{ml}$ and $4 / 76 \mathrm{mg} / \mathrm{ml}$ of these antibiotics respectively, and moderately resistance to cefotaxime $64.9 \%$, ceftriaxone $64.9 \%$ and chloramphenicol $64.9 \%$ with MIC $4 \mathrm{mg} / \mathrm{ml}$ for CTX and CRO at else, and MIC $8 \mathrm{mg} / \mathrm{ml}$ for C only. All isolates showed a sensitivity of $100 \%$ for each of Vancomycin and Linzolid with MIC $\leq 1 \mathrm{mg} / \mathrm{ml}$ and $\leq 2 \mathrm{mg} / \mathrm{ml}$, respectively. $S$. pneumoniae isolates showed a high rate of sensitivity to Ertapenem $97.3 \%$ with MIC $\leq 1 \mathrm{mg} / \mathrm{ml}$, Telithromycin $89.2 \%$ with MIC $\leq 1$, and Meropenem $86.5 \%$ with MIC $\leq 0.25$ $\mathrm{mg} / \mathrm{ml}$.

FLAMM ET AL. (2013) found that ceftriaxone MIC50/90, $\leq 0.25 / 2 \mu \mathrm{g} / \mathrm{mL}$ when tested against $S$. pneumoniae isolates in the USA during 20092012. There was a high rate of resistance to levofloxacin which exhibited high rates of susceptibility at $98.9 \%$. Ceftriaxone and amoxicillin/ clavulanate susceptibilities were $89.2 \%$ and $83.3 \%$, respectively. There was a high rate of resistance to erythromycin at $42.7 \%$, and resistance to tetracycline, trimethoprim/sulfamethoxazole and clindamycin ranged from $20.3-24.6 \%$. Only $86.5 \%$ of $S$. pneumoniae strains were inhibited at a penicillin MIC of $\leq 2 \mu \mathrm{g} / \mathrm{mL}$ (penicillin parenteral non-meningitis susceptible breakpoint) and $57.3 \%$ at $\leq 0.06 \mu \mathrm{g} / \mathrm{mL}$. 
Table 2. MIC values of S. pneumoniae isolates toward antibiotics

\begin{tabular}{|c|c|c|c|c|c|c|c|c|c|c|}
\hline \multirow[t]{2}{*}{ Antibiotics } & \multirow[t]{2}{*}{ Symbol } & \multicolumn{3}{|c|}{ Break point } & \multicolumn{2}{|c|}{$S$} & \multicolumn{2}{|c|}{ I } & \multicolumn{2}{|c|}{$\mathrm{R}$} \\
\hline & & $\mathrm{S} \leq$ & I & $\geq \mathrm{R}$ & No. & $(\%)$ & No. & $(\%)$ & No. & $(\%)$ \\
\hline Amoxicillin & AMX & 2 & 4 & 8 & 24 & 64.9 & 0 & 0 & 13 & 35.1 \\
\hline Benzylpenicillin & $\mathrm{P}$ & 2 & 4 & 8 & 10 & 27.1 & 15 & 40.5 & 12 & 32.4 \\
\hline Cefotaxime & CTX & 1 & 2 & 4 & 13 & 35.1 & 0 & 0 & 24 & 64.9 \\
\hline Ceftriaxone & CRO & 1 & 2 & 4 & 13 & 35.1 & 0 & 0 & 24 & 64.9 \\
\hline Chloramphenicol & $\mathrm{C}$ & 4 & - & 8 & 13 & 35.1 & 0 & 0 & 24 & 64.9 \\
\hline Ertapenem & ETP & 1 & 2 & 4 & 36 & 97.3 & 0 & 0 & 1 & 2.7 \\
\hline Erythromycin & E & 0.25 & 0.5 & 1 & 0 & 0 & 0 & 0 & 37 & 100 \\
\hline Levofloxacin & LEV & 2 & 4 & 8 & 20 & 54.1 & 4 & 10.8 & 13 & 35.1 \\
\hline Linezolid & LNZ & 2 & - & - & 37 & 100 & 0 & 0 & 0 & 0 \\
\hline Meropenem & MEM & 0.25 & 0.5 & 1 & 32 & 86.5 & 0 & 0 & 5 & 13.5 \\
\hline Moxifloxacin & MXF & 1 & 2 & 4 & 31 & 83.8 & 0 & 0 & 6 & 16.2 \\
\hline Ofloxacin & OFX & 2 & 4 & 8 & 31 & 83.8 & 0 & 0 & 6 & 16.2 \\
\hline Telithromycin & TEL & 1 & 2 & 4 & 33 & 89.2 & 0 & 0 & 4 & 10.8 \\
\hline Tetracycline & $\mathrm{TE}$ & 1 & 2 & 4 & 17 & 45.9 & 0 & 0 & 20 & 54.1 \\
\hline $\begin{array}{l}\text { Trimethoprim/ } \\
\text { Sulfamethaxzol }\end{array}$ & SXT & $0.5 / 9.5$ & $\begin{array}{l}1 / 1 \\
4 / 7\end{array}$ & $9-2 / 38$ & 6 & 16.2 & 0 & 0 & 31 & 83.8 \\
\hline Vancomycin & VA & 1 & - & - & 37 & 100 & 0 & 0 & 0 & 0 \\
\hline
\end{tabular}

RIJAL ET AL. (2010) found that MICs against penicillin, Chloramphenicol, Erythromycin and Cefotaxime were determined. Whereas penicillin MICs were mostly around $0.016 \mathrm{mgl}^{-1}$ and showed $3(6.5 \%)$ were intermediately resistant, no penicillin resistant strains were isolated. Over the 4 years, erythromycin MICs ranged from 0.062 to $8 \mathrm{mgl}^{-1}$ and resistance was recorded in one $(2.17 \%)$. Chloramphenicol MICs ranged from 1 to $4 \mathrm{mgl}^{-1}$ and overall one $(2.17 \%)$ was considered resistant towards Chloramphenicol whereas the MICs of Cefotaxime ranges from $0.008 \mathrm{mgl}^{-1}$ to $0.25 \mathrm{mgl}^{-1}$. Overall only one $2.17 \%$ ) was found to be intermediately resistant to Cefotaxime in this study period.

Forty-six strains (72\%) were susceptible to penicillin with a minimum inhibitory concentration (MIC) $<0.06 \mu \mathrm{g} / \mathrm{mL}$. Among the resistant strains, $20 \%$ showed intermediate resistance (between 0.12 and $1 \mu \mathrm{g} / \mathrm{mL}$ ) and $8 \%$ showed full resistance $(>2 \mu \mathrm{g} / \mathrm{mL})$. These results showed that the local and national rates of penicillin resistance are stable compared to the results of other investigators, who reported rates of 26\% (ZETTLER ET AL., 2006), and can still be considered a good option to control pneumococcal infection.

\section{References}

Al-Taaie M.J.K. 2013. Bacterial Causes of Typical Pneumonia and Immune Response of Laboratory Animals for Some Bacterial Spp. Isolated from Pneumonic Patients in
Babylon Province. M. Sc. Thesis of Microbiology. College of Medicine. Univ. of Babylon.

Balaban N.Q., Merrin, J., Chait R., Kowalik L., Leibler S. 2004. Bacterial persistence as a phenotypic switch. Science, 305: 1622-1625.

Bauer A.W., Kirby W.M.M., Sherris J.S., Turk M. 1966. Antibiotic susceptibility testing by a standardized single disk method. Amer. J. Clinic. Pathol., 45:493-496.

Black R.E., Cousens S., Johnson H.L. Lawn J.E., Rudan I., Bassani D.G. et al. 2010. Child Health Epidemiology Reference Group of WHO and UNICEF. Global, regional, and national causes of child mortality in 2008: a systematic analysis. Lancet, 375: 1969-1987.

Bryce J., Boschi-Pinto C., Shibuya K., Black R.E. 2005. WHO estimates of the causes of death in children. Lancet, 365 : 1147-1152.

Clinical and Laboratory Standards Institute. Performance Standards for Antimicrobial Susceptibility Testing: TwentyFourth Informational Supplement M02-A11, M07-A9, and M11-A8. CLSI, (2014). Wayne, PA, USA.

Collee J.G., Fraser A.G., Marmion B.P., Simpson S.A. 1996. Mackie and McCartney. Practical medical microbiology. $14^{\text {th }}$ ed. Churchill Livingstone inc., USA.

Domenech A., Ardanuy C., Calatayud L., Santos S., Fe Tubau G.I., Verdaguer R., Dorca J., Pallares R., Martin R., Linăres J. 2011. Serotypes and genotypes of Streptococcus pneumoniae causing pneumonia and acute exacerbations in patients with chronic obstructive pulmonary disease. J. Antimicrob Chemother. (2011) 66 (3): 487-493. DOI: https://doi.org/10.1093/jac/dkq480

Flamm R.K., Sader H.S., Jones R.N. 2013. Antimicrobial Activity of Ceftaroline Tested against Multidrug Resistant (MDR) Streptococcus pneumoniae in the USA (2009-2012). IDWEEK. 893.

Fuller J.D., Low D.E. 2005. A review of Streptococcus pneumoniae infection treatment failures associated with fluoroquinolone resistance. Clin Infect Dis, 41: 118-21. 
Gentile A., Bardach A., Ciapponi A., Garcia-Marti S., Aruj B., Glujovsky D.B., Calcagno B., Mazzoni A.P., Colindres R.E. 2012. Epidemiology of community-acquired pneumonia in children of Latin America and the Caribbean: a systematic review and meta-analysis. Int. J. Infect. Dis., 16: 5-15.

Gossens H. 2009. Antibiotic consumption and link to resistance. Clin. Microbiol. Infec., 15(suppl.3), 12-15.

Guido F., Pascale F. 2005. Performance of the New VITEK 2 GP Card for Identification of Medically Relevant GramPositive Cocci in a Routine Clinical Laboratory. J. Clin Microbiol., 43(1): 84-88.

Gupta D., Agarwal R., Aggarwal A.N., Singh N., Mishra N., Khilnan G.C., Samaria J.K., Gaur S.N., Jindal S.K. 2012. Guidelines for diagnosis and management of Communityacquired pneumonia in adult. Lung India (Supplement 2): 33.

Iwalokun B.A., Fowora M., Akinloye O., Oluwadun A., Antonio M., Adegbola R.A. 2012. A retrospective study of clinical Streptococcus pneumoniae isolates from four health facilities in South-West Nigeria. Int. J. Medicine and Medical Sci., 4(8): $160-170$.

Jorgensen J.H., Crawford S.A., McElmeel L.M., Whitney C.G. 2004. Detection of resistance to gatifloxacin and moxifloxacin in Streptococcus pneumoniae with the VITEK 2 instrument. J. Clin. Microbiol., 42: 5928-5930.

Kanj S.S., El-Dbouni O., Kanafani Z.A., Araj G.F. 2007. Antimicrobial susceptibility of respiratory pathogens at the American University of Beirut Medical Center. Int. J. Infect. Dis., 11: 554-556.

Levin A.S., Teixeira L.M., Sesselogo J.F., Barone A.A. 1996. Resistance of Streptococcus pneumoniae to antimicrobials in São Paulo, Brazil: clinical features and serotypes. Rev. Inst. Med. Tropical, 38(3): 187-192.

Levy S.B., Marshall B. 2004. Antibacterial resistance worldwide: causes, challenges and responses. Nat. Med., 10: S122-S129.

Macfaddin J.F. 2000. Biochemical tests for identification of medical Bacteria. 3rd-ed, William and Wilkins, U.S.A.

Maruyama T., Gabazza E.C., Morser J., Takagi T.D., AlessandroGabazza C. 2010. Community-acquired pneumonia and nursing home-acquired pneumonia in the very elderly patients. Respir Med., 104: 584-592.

O’Brien K.L., Wolfson L.J., Watt J.P., Henkle E., Deloria-Knoll M. 2009. Burden of disease caused by Streptococcus pneumoniae in children younger than 5 years: global estimates. Lancet, 374: 893-902.
Obaro S.K. 2000. Confronting the pneumococcus: a target shift or bullet change? Vaccine, 19: 1211-1217.

Paul J. 1997. HIV and pneumococcal infection in Africa. Microbiological aspects. Trans. R. Soc. Trop. Med. Hyg., 91: 632-637.

Pejcic T., Dordevic I., Stankovic I., Borovac D.N., Petkovic T.R. 2011. Prognostic mortality factors of community-acquired pneumonia in the elderly. Sci. J. Fac. Med. in Nis., 28(2): 71-76.

Riedel S., Beekmann S.E., Heilmann K.P., Richter S.S. GarciadeLomas J., Ferech M., Goosens H., Doern G.V. 2007. Antimicrobial use in Europe and antimicrobial resistance in Streptococcus pneumoniae. Eur. J. Clin. Microbiol. Infect. Dis., 26: 485-490.

Rijal B., Tandukar S., Adhikari R., Tuladhar N.R., Sharma P.R., Pokharel B.M., Gami F.C., Shah A., Sharma A., Gauchan P., Sherchand J.B., Burlakoti T., Upreti H.C., Lalitha M.K., Thomas K., Steinhoff M. 2010. Antimicrobial susceptibility pattern and serotyping of Streptococcus pneumoniae isolated from Kanti Children Hospital in Nepal. Kathmandu Univ. Med. J., 30, 8(2): 164-168.

Sener B., Tunckanat F., Ulusoy S., Tünger A., Söyletir G., Mülazlmoğlu L. 2007. A survey of antibiotic resistance in Streptococcus pneumoniae and Haemophilusinfluenzae in Turkey, 2004-2005. J. Antimicrob. Chemother., 60: 587-593.

Tran T.D.H., Hyog-Young K., Kim E., Kim K., Briles D.E., Pyo S., Rhee1 D. 2011. Decrease in Penicillin Susceptibility Due to Heat Shock Protein ClpL in Streptococcus pneumoniae. Antimicrob. Agents Chemother., 55(6): 2714-2728. American Society for Microbiology.

Weber F.T., Dias C., da Costa M. 2010. Antimicrobial susceptibility of Streptococcus pneumoniae and genotypic characterization of erythromycin-resistant strains in Porto Alegre, Brazil. Brazilian J. Microbiology, 41: 1-5. DOI: $10.1590 /$ S1517-83822010000100001

Wilson M.J.B., Martin D.E. 1972. Quantitative sputum culture as a means of excluding false positive reports in the routine micrpbiology laboratory. J. Clin. Path., 25: 697700.

Zettler E.W., Scheibe R.M., Dias C.A.G., Santafé P., Santos D.S., Moreira J.D. Fritsher C.C. 2006. Determination of penicillin resistance in Streptococcus pneumoniae isolates from Brazil by PCR. Intern. J. Infect. Dis., 10: 110-115. 